\title{
Study of Structure of Li- and Mn-rich Transition Metal Oxides Using 4D-STEM
}

\author{
Alpesh Khushalchand Shukla ${ }^{1}$, Colin Ophus ${ }^{2}$, Christoph Gammer ${ }^{3}$ and Quentin Ramasse \\ 1. \\ SuperSTEM, Daresbury, United Kingdom \\ 2. \\ National Center of Electron Microscopy, Molecular Foundry, Lawrence Berkeley National Laboratory, \\ Berkeley, United States. \\ University of Vienna, Vienna, Austria.
}

The structure of $\mathrm{Li}$ - and $\mathrm{Mn}$-rich transition metal oxides $\left(\mathrm{Li}_{1+\mathrm{x}} \mathrm{M}_{1-\mathrm{x}} \mathrm{O}_{2}\right.$, where $\mathrm{M}$ is usually a combination of transition metals such as Mn, Co and Ni, called LMRTMO henceforth) has been debated extensively for the past several years. It has been recently shown, by imaging entire primary particles at atomic resolution at multiple zone axes using high angle annular dark field (HAADF-) scanning transmission electron microscopy (STEM), that the bulk of the oxides can be described as an aperiodic crystal consisting of randomly stacked domains that correspond to three variants of monoclinic structure [1] as shown in Figure 1 (a). Using HAADF-STEM, it was demonstrated that the particles did not contain two phases (trigonal and monoclinic) in the bulk as described earlier [2], since the size of the primary particles was small enough show that the monoclinic phase was the only phase present in entire particles. However, larger sized particles are often preferred for the cathode materials in order to obtain better volumetric energy density. For these applications, imaging entire particles at atomic resolution is very difficult and in some cases impossible owing to regions of higher thickness. In this paper we demonstrate the use of 4D-STEM [3] using large fields of view on a commercial cathode material to confirm that the bulk of the primary particles is made up of a single phase and consists of domains corresponding to three variants of monoclinic phase.

The structure of TODA HE5050, a commercial cathode material having composition $\mathrm{Li}_{1.2}\left(\mathrm{Ni}_{0.15} \mathrm{Mn}_{0.55} \mathrm{Co}_{0.1}\right) \mathrm{O}_{2}$ was first determined using a multiple technique approach described elsewhere [1], principally by HAADF-STEM imaging using TEAM 0.5 microscope operated at $80 \mathrm{kV}$ and Nion UltraSTEM microscope operated at $100 \mathrm{kV}$. 4D-STEM datasets were obtained using an FEI Titan microscope operated at $120 \mathrm{kV}$. A Digital Micrograph script was used to collect the 4D STEM data as described in reference [4] covering the entire particle using a step size of $5 \mathrm{~nm}$. Higher resolution scans that partially covered the particle were also recorded using a step size of $2 \mathrm{~nm}$. Figure 2 (b) shows a the summed CBED pattern obtained using [100] $]_{\text {supercell }}$ zone axis. The streaks shown in Figure 2 (b) are observed due to the closeness of reflections corresponding to the three variants of the monoclinic phase, namely, [100], [1-10] and [110]. A convergence angle of 0.54 radians was used that minimized the overlap between the discs corresponding to the reflections from aforementioned variants.

Virtual apertures were used to separate the reflections from these three variants as shown in Figure $2 \mathrm{c}$. Figure $2 \mathrm{~d}$ shows the resulting reconstructed virtual dark field image that clearly shows the presence of domains corresponding to the three variants of monoclinic phase. With a single selected area electron diffraction pattern covering the entire particle, it would not be possible to differentiate if the structure consists of a single monoclinic phase or a combination of monoclinic and trigonal phases, since the fundamental reflections in the diffraction patterns are common between both structures. By rastering the beam over the entire particle we demonstrated that CBED pattern corresponding to the monoclinic phase was found over the entire particle. We were also able to demonstrate the presence of surface spinel that 
has been previously shown using atomic resolution HAADF STEM imaging [1], in spite of the fact that the surface is only about $2 \mathrm{~nm}$ in thickness. In this example, a $230 \mathrm{~nm}$ sized equiaxed particle was used to demonstrate the application of 4D-STEM on a complex battery material and the technique can conveniently be used even on larger, micron-sized particles and can be particularly useful for studying electrochemically cycled samples, whereby atomic resolution imaging could become increasingly difficult as the battery is cycled, owing to the resulting phase transformations and misorientations introduced into the particles [5].

\section{References:}

[1] A. K. Shukla, et al, Nature Communications, 6 (2015) p. 8711.

[2] M. M. Thackeray et al, Electrochemistry Communications, 8(6), (2006) p. 1531-1538.

[3] C. Ophus, et al, Microsc Microanal, 20(3) (2014), pp. 62-63.

[4] C. Gammer, et al, Ultramicroscopy, Ultramicroscopy, 55 (2015) , pp. 1-10.

[5] The authors acknowledge support of the Molecular Foundry, Lawrence Berkeley Lab, which is supported by the U.S. Department of Energy under Contract \# DE-AC02-05CH11231 and also the support from SuperSTEM, Daresbury, UK, which is the National Facility for Aberration- Corrected Scanning Transmission Electron Microscopy, supported by the Engineering and Physical Science Research Council.
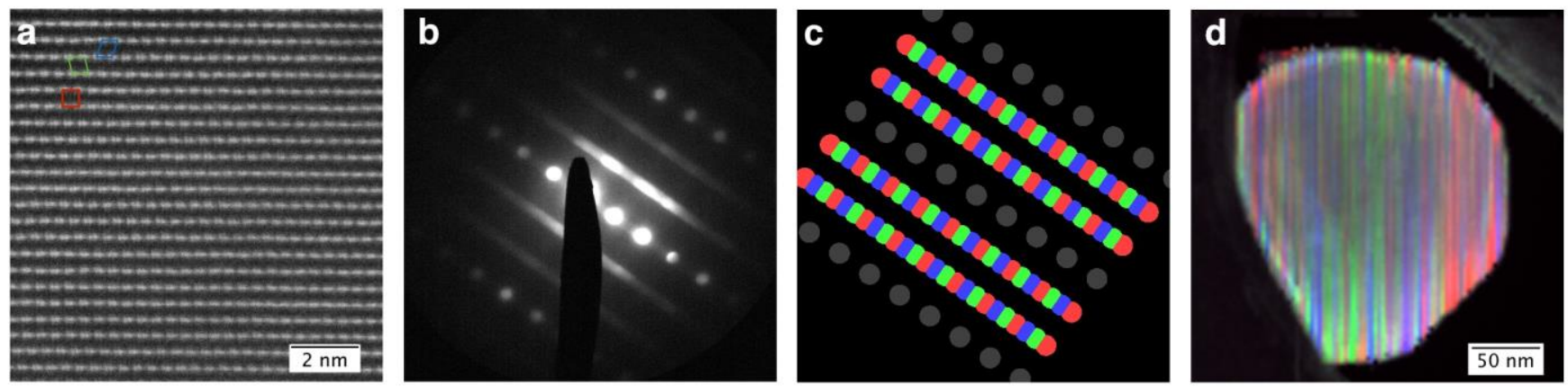

Figure 1. (a) HAADF STEM image of LRTMO cathode particle (b) Summed CBED pattern from the 4D STEM dataset, (c) location and shape of virtual apertures corresponding to the three variants and (d) Reconstructed virtual dark field image showing the domains corresponding to the three monoclinic variants in red, blue and green. 